\title{
Prognostic values of transketolase family genes in ovarian cancer
}

\author{
MENGHUANG ZHAO, MIAOMIAO YE, JUNHAN ZHOU and XUEQIONG ZHU \\ Department of Obstetrics and Gynecology, The Second Affiliated Hospital of Wenzhou Medical University, \\ Wenzhou, Zhejiang 325027, P.R. China
}

Received January 19, 2019; Accepted August 1, 2019

DOI: $10.3892 / \mathrm{ol} .2019 .10818$

\begin{abstract}
Transketolase genes are key rate-limiting enzymes in the non-oxidative part of the pentose phosphate pathway, which is an important metabolic pathway in ribose-5-phosphate production. Three human transketolase genes have been identified: Transketolase (TKT), transketolase-like gene 1 (TKTL1) and transketolase-like gene 2 (TKTL2). Transketolase genes serve crucial roles in the tumorigenesis, metastasis and outcome of multiple types of cancer. However, the expression levels and prognostic values of transketolase family genes in patients with ovarian cancer remain unclear. The purpose of the study was to analyze the expression level and prognostic significance of transketolase family genes in ovarian cancer. In the present study, the mRNA expression levels of three transketolase genes in ovarian cancer and normal ovarian tissue were compared by Oncomine. The prognostic values of these genes were systemically assessed using the Kaplan-Meier plotter database. In addition, the associations between the mRNA levels of these transketolase genes and the clinicopathological characteristics of patients with ovarian cancer, such as histological subtype, clinical stage, grade, tumor protein p53 (TP53) mutation status and chemotherapy history were studied. The prognostic roles of transketolase genes were also evaluated in a validation dataset. The results demonstrated that TKT and TKTL1 expression in ovarian cancer tissues was elevated compared with that in normal ovarian tissues. In addition, high mRNA expression of the three transketolase genes was identified to be associated with poorer progression-free survival (PFS) in patients with serous ovarian cancer, especially in patients at an advanced stage. TKTL2 was significantly associated with poor overall survival in all patients with ovarian cancer. Additionally, transketolase family genes served a role in predicting PFS in patients with ovarian cancer treated with platinum and/or taxol. High
\end{abstract}

Correspondence to: Professor Xueqiong Zhu, Department of Obstetrics and Gynecology, The Second Affiliated Hospital of Wenzhou Medical University, 109 Xueyuan Xi Road, Wenzhou, Zhejiang 325027, P.R. China

E-mail: zjwzzxq@163.com

Key words: ovarian cancer, transketolase, prognosis, Kaplan-Meier plotter, expression expression of the three transketolase genes was associated with unfavorable PFS in patients with TP53-mutated ovarian cancer, but not in patients with TP53 wild-type ovarian cancer. These results suggested that transketolase family genes may serve important roles in the prognosis of patients with ovarian cancer.

\section{Introduction}

Ovarian cancer is the most lethal gynecologic cancer, usually bearing a poor outcome (1). In 2019, a total of 22,530 new ovarian cancer cases and 13,980 ovarian cancer-associated mortalities were predicted to occur in the United States (1). Although aggressive surgery and combination chemotherapy have improved survival, the 5-year overall survival (OS) rate remains only $30 \%$ (2). Thus, identification of prognostic markers is of great need to allow improved risk prediction.

A metabolic hallmark of the majority of cancer cells is the shift from oxidative phosphorylation to aerobic glycolysis even with abundant oxygen, which is known as the Warburg effect (3). Since inhibiting the entry of pyruvate into the mitochondria leads to the redirection of glycolytic intermediates into the pentose phosphate pathway, which provides precursors for the synthesis of nucleotides and lipids, the Warburg effect contributes to the highly anabolic phenotype of cancer (4). Therefore, elevated expression of key enzymes that catalyze the pentose phosphate pathway has been observed in several types of cancer, including hepatocellular cancer (5) and colorectal carcinoma (6). The pentose phosphate pathway is responsible for the production of ribose-5-phosphate and NADPH (7). Ribose-5-phosphate is necessary for the synthesis of nucleic acids to meet the needs of rapidly proliferating tumor cells (8). The pentose phosphate pathway comprises two branches, oxidative and non-oxidative. The non-oxidative branch of the pentose phosphate pathway provides $>85 \%$ of ribose for nucleic acids synthesis of certain tumor cells, including pancreatic adenocarcinoma (9). The transketolase family genes encode thiamine-dependent enzymes, which are key enzymes in the non-oxidative part of the pentose phosphate pathway (10). The transketolase family comprises three human genes, transketolase (TKT) and TKT-like genes 1 and 2 (TKTL1 and TKTL2) (11).

Previous studies have revealed that the transketolase family genes serve important roles in the initiation, progression and cross-talk with other signaling pathways in cancer. Upregulated transketolase family genes have been identified 
in multiple types of cancer, including hepatocellular carcinoma (5), esophageal cancer (12), lung cancer (13), head and neck cancer (14), and colorectal carcinoma (6) and endometrial carcinoma (15). Overexpression of these genes is associated with poor outcome of patients with esophageal (16), lung (17), gastric (18), breast (19), colon and urothelial (20) cancer. Aberrant expression of transketolase family genes has also been observed in ovarian cancer (21). However, studies on the prognostic value of the transketolase family genes in ovarian cancer are limited (22). In addition, none of these studies have comprehensively assessed the prognostic effects of individual transketolase family components in ovarian cancer at the mRNA level. The aim of the present study was to determine the expression levels and prognostic significance of transketolase family genes in patients with ovarian carcinoma.

\section{Materials and methods}

Datasets. Oncomine is an online collection of datasets with data mining platform (23). The expression of three transketolase family genes (TKT, TKTL1 and TKTL2) in normal ovarian tissues and ovarian cancer were analyzed by Oncomine. Bonome, Lu, Hendrix and the Cancer Genome Atlas cancer (TCGA) ovarian datasets were analyzed in the present study.

Survival analysis. Kaplan-Meier plotter (24) is a widely used online database that integrates gene expression and clinical data of various types of cancer such as breast (24), lung (25) and ovarian $(26,27)$ cancer. In the present study, the progression-free survival (PFS) of 1,435 patients and OS of 1,656 patients with ovarian cancer were assessed using the Kaplan-Meier plotter database (www.kmplot.com). The information about gene expression and survival of patients with ovarian cancer was collected from the Gene Expression Omnibus (GEO), Cancer Biomedical Informatics Grid (http://cabig.cancer.gov/) and TCGA cancer datasets (28). Clinical data, including stage, histology, grade, tumor protein p53 (TP53) mutation status and treatment, were also obtained. The association between transketolase gene mRNA levels and clinicopathological parameters was determined in patients with ovarian cancer with the corresponding clinical information. Transketolase family genes TKT, TKTL1 and TKTL2 were entered into the database to produce Kaplan-Meier survival plots. Patients were classified into 'low' and 'high'-expression groups according to the quantile expression of the proposed biomarker. Hazard ratio (HR) with $95 \%$ confidence intervals (CI) and log-rank P-value were calculated.

Validation dataset. The GSE9891 dataset, which was part of the Kaplan-Meier plotter, was downloaded separately from GEO as a validation dataset (29). Expression data and clinic information of patients with ovarian cancer from GSE9891 were used to validate the prognostic values of transketolase family genes in ovarian cancer.

Statistical analysis. Unpaired Student's-test was performed to compare the expression level of transketolase family genes between ovarian cancer and normal ovarian tissues from Oncomine. Results were presented in box plots. Kaplan-Meier curve was used to assess the association between expression of transketolase family genes and the survival rate in ovarian cancer. The log-rank test was used to compare the survival differences between the low and high-expression groups from the Kaplan-Meier plotter. $\mathrm{P}<0.05$ was considered to indicate a statistically significant difference.

\section{Results}

Transcription level of TKT in ovarian cancer compared with that in normal tissues. The transketolase family involves three human genes: TKT, TKTL1 and TKTL2. The differential expression of these three genes between normal ovarian tissue and ovarian cancer was analyzed using the Oncomine database. In the Bonome dataset, which comprised 185 ovarian cancer tissues and 10 normal ovarian tissues from patients with other gynecological diseases, the mRNA level of TKT in ovarian cancer tissue was higher compared with that in normal tissue (Fig. 1A). In the TCGA dataset, which comprised 586 serous ovarian cancer samples and 8 adjacent normal ovarian tissues from patients with ovarian cancer, no difference in TKT expression was observed between serous ovarian cancer and normal ovarian tissue (Table I). Since other types of ovarian cancer were rare, TKT expression was systematically analyzed by combining the Lu and Hendrix datasets, in which ovarian cancer cases were classified by histological types. The results of the analysis demonstrated that TKT was upregulated in endometrioid, clear cell and mucinous ovarian cancer compared with its expression in normal ovarian tissue (Table I).

Transcription level of TKTL1 in ovarian cancer compared with that in normal tissues. Compared with that in normal ovarian tissue, TKTL1 expression was increased by 1.398 -fold in all ovarian cancer samples in the Bonome dataset (Fig. 1B). According to the TCGA dataset, TKTL1 expression was increased by 2.384 -fold in serous ovarian cancer compared with that in normal ovarian tissue $(\mathrm{P}<0.001$; Table I). However, no difference in TKTL1 expression was observed between endometrioid, clear cell or mucinous ovarian cancer and normal ovarian tissue (Table I).

Transcription level of TKTL2 in ovarian cancer compared with that in normal tissues. No dataset compared TKTL2 expression between all ovarian cancer and normal ovarian tissues. Only the Lu dataset that separated ovarian cancer cases into different histological types was accessible in Oncomine; no significant differences in TKTL2 expression levels were observed between serous, endometrioid, clear cell or mucinous ovarian cancer and normal ovarian tissue (Table I).

Prognostic value of TKT in ovarian cancer. The prognostic value of transketolase family genes was assessed. Survival information for the three transketolase genes was obtained from the Kaplan-Meier plotter. The prognostic value of TKT mRNA expression in ovarian cancer was evaluated; the Affymetrix identifier (ID) was 205168_at TKT. PFS curves were plotted for all patients with ovarian cancer $(\mathrm{N}=1,435$; Fig. 2A), patients with serous cancer ( $N=1,104$; Fig. 2B) and endometrioid cancer ( $\mathrm{N}=51$; Fig. 2C). Increased expression 
A

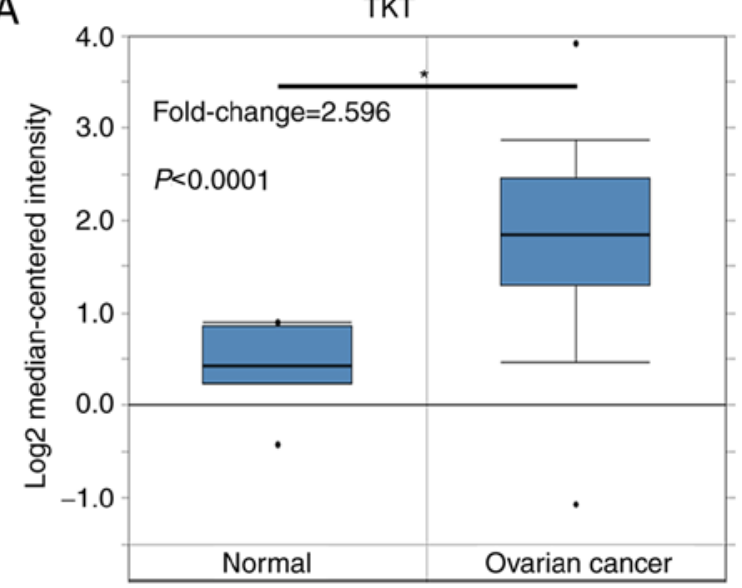

B

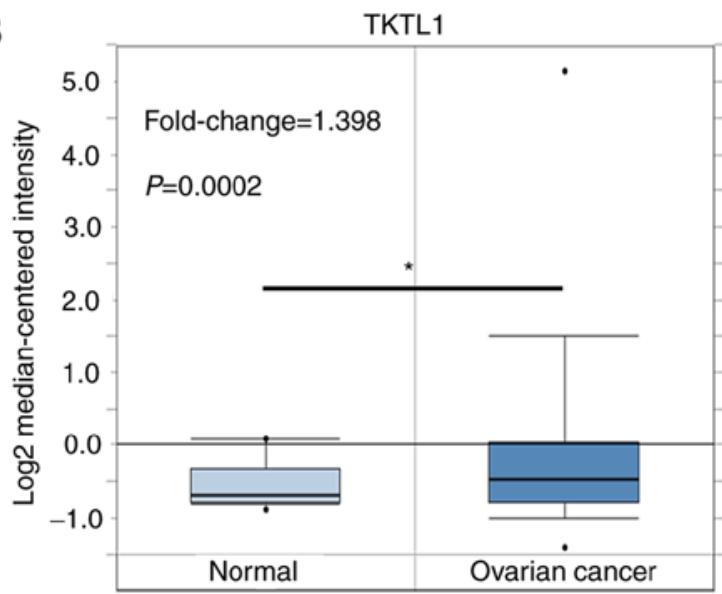

Figure 1. Comparison of the expression profile of TKT and TKTL1 in normal ovarian tissue vs. ovarian cancer tissue. (A) TKT. (B) TKTL1. * $<0.05$. TKT, transketolase; TKTL1, TKT-like 1.

levels of TKT mRNA were identified to be associated with poorer PFS in all patients with ovarian cancer (HR, 1.25; 95\% CI, 1.10-1.42; $\mathrm{P}=0.0007)$. High TKT expression was also associated with poor PFS in serous ovarian cancer (HR, 1.27; 95\% CI, 1.10-1.47; P=0.0010). However, TKT mRNA expression levels were not associated with PFS in patients with endometrioid cancer. The OS between the high and low-TKT expression groups in all ovarian cancer and serous ovarian cancer cases was not significantly different (Fig. 3A and B). TKT mRNA expression was not associated with OS in patients with endometrioid cancer (Fig. 3C).

Prognostic value of TKTL1 in ovarian cancer. The prognostic value of TKTL1 mRNA expression in ovarian cancer was evaluated; the Affymetrix ID was 216370_s_at TKTL1. No differences were observed in PFS between the high and low-TKTL1 expression groups in all patients with ovarian cancer (Fig. 4A). However, stratified analyses demonstrated that high expression of TKTL1 was associated with poor PFS in patients with serous ovarian cancer (HR, 1.27; 95\% CI, 1.08-1.50; $\mathrm{P}=0.0036$; Fig. 4B), as well as in patients with endometrioid ovarian cancer (HR, 4.33; 95\% CI, 0.99-18.88; $\mathrm{P}=0.0340$; Fig. 4C). TKTL1 mRNA expression levels were not associated with OS in all patients with ovarian cancer (Fig. 5A), nor in patients with serous ovarian cancer (Fig. 5B) or endometrioid ovarian cancer (Fig. 5C).

Prognostic value of TKTL2 in ovarian cancer. The prognostic value of TKTL2 mRNA expression in ovarian cancer was evaluated; the Affymetrix ID was 223829_at TKTL2. High TKTL2 mRNA expression indicated unfavorable PFS for all patients with ovarian cancer (HR, 1.49; 95\% CI, 1.19-1.85; $\mathrm{P}=0.0004$; Fig. 6A) and patients with serous ovarian cancer (HR, 1.44; 95\% CI, 1.13-1.84; P=0.0031; Fig. 6B). However, TKTL2 mRNA expression levels were not associated with PFS in patients with endometrioid ovarian cancer (Fig. 6C). High expression of TKTL2 was associated with OS in all patients with ovarian cancer (HR, 1.38; 95\% CI, 1.07-1.76; $\mathrm{P}=0.0110$; Fig. 7A). TKTL2 exhibited no association with OS in patients with serous ovarian cancer (Fig. 7B) or endometrioid ovarian cancer (Fig. 7C).
Prognostic value of transketolase family genes in different subtypes of ovarian cancer. The association between individual transketolase gene mRNA expression levels and clinicopathological features of patients with ovarian cancer was analyzed. Only samples with the relevance clinical information were included. The association with pathological clinical stage (Table II), grade (Table III), chemotherapy treatment (Table IV) and TP53 mutation status (Table V) of patients with ovarian cancer was determined. As presented in Table II, the three transketolase genes were associated with poor PFS in patients with stage III/IV ovarian cancer, but not stage I/II ovarian cancer. The individual transketolase genes were associated with poor PFS in patients with grade I/II and grade III ovarian cancer (Table III). TKT and TKTL2 were associated with unfavorable PFS in patients with ovarian cancer treated with platinum chemotherapy (Table IV). In addition, high levels of TKTL1 and TKTL2 were associated with poor PFS in patients with ovarian cancer treated with taxol chemotherapy (Table IV). The association between transketolase family gene expression levels and PFS of ovarian cancer patients was also evaluated based on the TP53 mutation status; the individual transketolase genes were only significantly associated with poor PFS in patients with TP53-mutated ovarian cancer, but not in those with TP53 wild-type ovarian cancer.

Validation of prognostic values of transketolase family genes in ovarian cancer. For the verification of the prognostic roles of transketolase family genes, the GSE9891 dataset was analyzed by log-rank test (Fig. 8). Similar results to the Kaplan-Meier plotter analysis were observed: In all patients with ovarian cancer, high expression levels of TKT and TKTL2 were associated with poor PFS, whereas TKTL1 was not associated with PFS.

\section{Discussion}

Transketolases, which are key enzymes of the non-oxidative branch of the pentose phosphate pathway, are associated with the tumorigenesis and development of a variety of tumors (10). The aim of the current study was to investigate the expression levels and prognostic values of transketolase genes in ovarian cancer. 
Table I. Comparison of the expression profile of transketolase family genes in normal ovarian tissue vs. various types of ovarian cancer.

Tissues, $\mathrm{n}$

Transketolase family gene

Cancer type

Normal

Cancer

P-value

TKT

Serous 8

Endometrioid 9

Clear cell 9

Mucinous 9

586

0.409

Mucinous

46

46

15

Serous 8

22

$<0.001^{\text {a }}$ (upregulated)

TKTL1

Endometrioid

8

586

Clear cell

9

46

$0.007^{\mathrm{a}}$ (upregulated)

Mucinous

9

15

$0.001^{\text {a }}$ (upregulated)

Mucinous 9

TKTL2

Serous

Endometrioid

Clear cell

Mucinous

922

22

$<0.001^{\text {a }}$ (upregulated)

0.164

520

0.463

0.324

20

0.311

5

0.113

5

0.066

5

7

9

0.147

aP $<0.05$. TKT, transketolase; TKTL1, TKT-like 1; TKTL2, TKT-like 2.

A

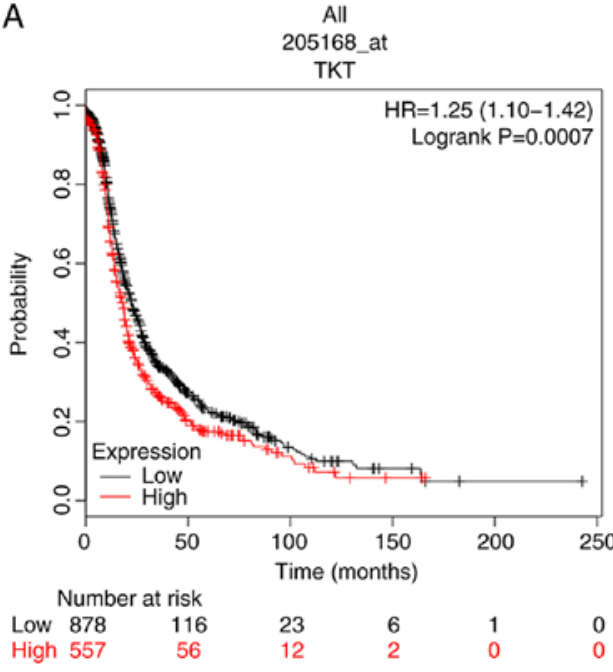

B
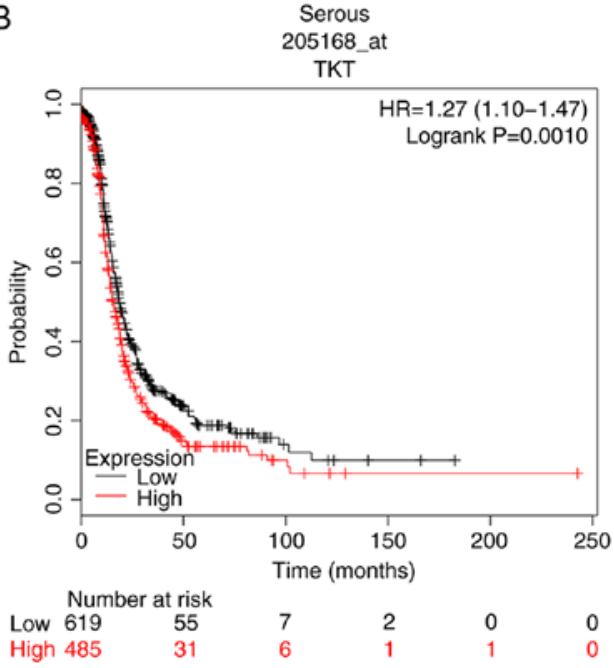

C

Endometrioid 205168_at

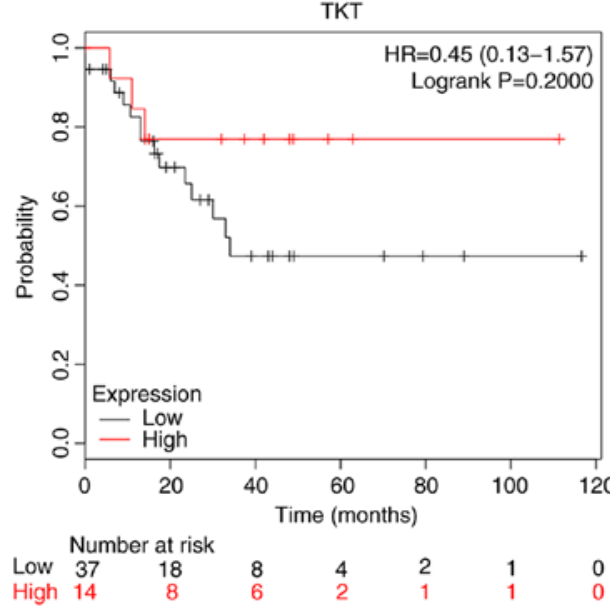

Figure 2. Prognostic value of TKT expression for PFS of patients with ovarian cancer based on the Kaplan-Meier plotter. Affymetrix identifier, 205168_at TKT. (A-C) PFS curves for (A) all patients $(\mathrm{N}=1,435)$, (B) patients with serous cancer $(\mathrm{N}=1,104)$ and (C) patients with endometrioid cancer $(\mathrm{N}=51)$. TKT, transketolase; PFS, progression-free survival. 
A

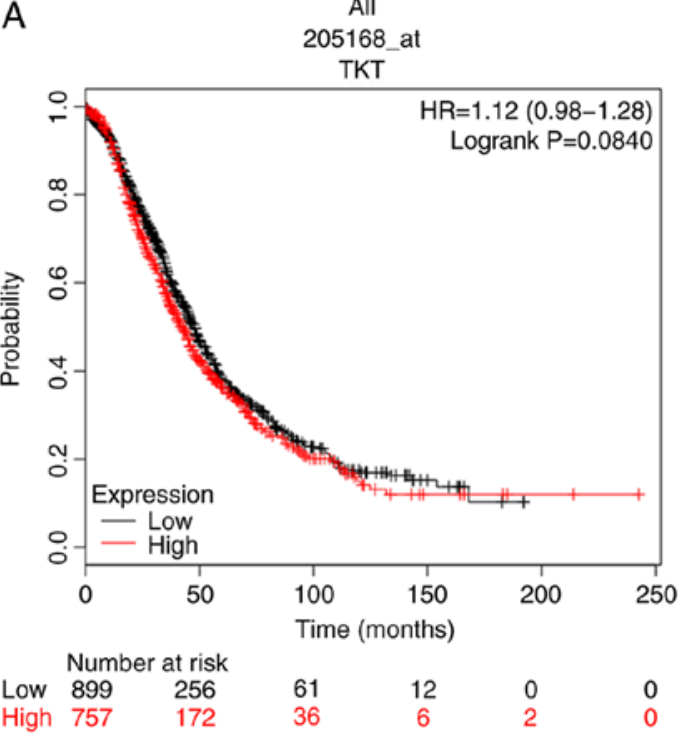

B

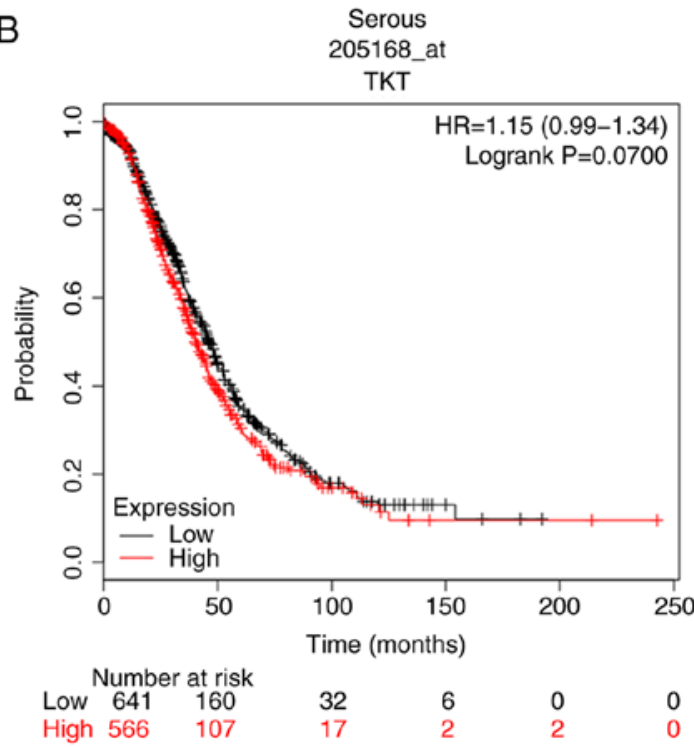

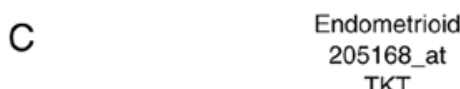

Endometrioid
205168_at

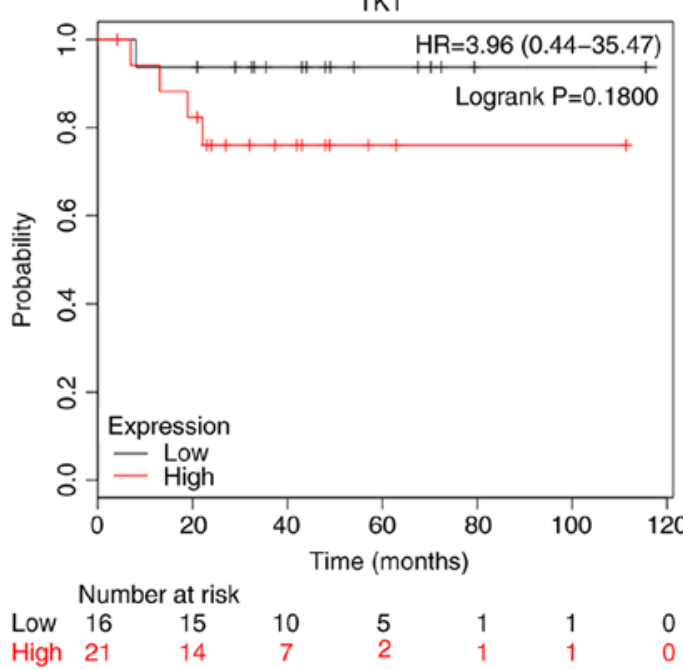

Figure 3. Prognostic value of TKT expression for OS of patients with ovarian cancer based on the Kaplan-Meier plotter. Affymetrix identifier, 205168_at TKT. (A-C) OS curves for (A) all patients $(\mathrm{N}=1,656),(\mathrm{B})$ patients with serous cancer $(\mathrm{N}=1,207)$ and $(\mathrm{C})$ patients with endometrioid cancer $(\mathrm{N}=37)$. TKT, transketolase; OS, overall survival.

TKT is not only involved in cancer development and progression, but also contributes to patient survival. For example, TKT has been reported to be upregulated in hepatocellular carcinoma (5). In addition, high TKT expression levels indicate poor survival in hepatocellular carcinoma and esophageal cancer $(5,16)$. A number of previous studies have indicated carcinogenic roles for TKT in breast cancer and gynecological tumors. In breast cancer, Tseng et al (30) demonstrated that TKT expression levels were upregulated in breast cancer compared with those in normal tissue, and were higher in metastatic lymphoid tissue compared with those in the primary site. Further analysis of the association between TKT expression and clinical parameters identified that TKT expression was associated with breast cancer stage and grade, patient age, and tumor size and type; in addition, patients with high TKT expression exhibited a worse outcome compared with that observed in the low-TKT expression group (30). A study by Yang et al (31) revealed that TKT was highly expressed in $81.1 \%$ of cervical cancer samples and was significantly upregulated in cervical cancer compared with its expression in normal cervical tissue. Chen et al (32) compared serum peptide profiles among healthy control subjects and patients with cervical cancer prior and subsequent to surgery. The results demonstrated that TKT expression levels were higher in patients with cervical cancer prior to surgery compared with those in healthy subjects and patients following surgery. In addition, TKT is expressed in ascites fluid from patients with ovarian cancer, but not from individuals with benign lesions (21). Yi et al (33) identified TKT in exosomes derived from two late-stage ovarian cancer cell lines and suggested that TKT may serve a dominant role in exosome-mediated carcinogenicity and cancer progression. Depletion of TKT by TKT siRNA inhibited proliferation in ovarian cancer cells in vitro (22). Oxythiamine, a transketolase inhibitor, also decreased tumor cell proliferation in ovarian cancer cell lines and primary serous ovarian cancer cells (22). Only one 
A

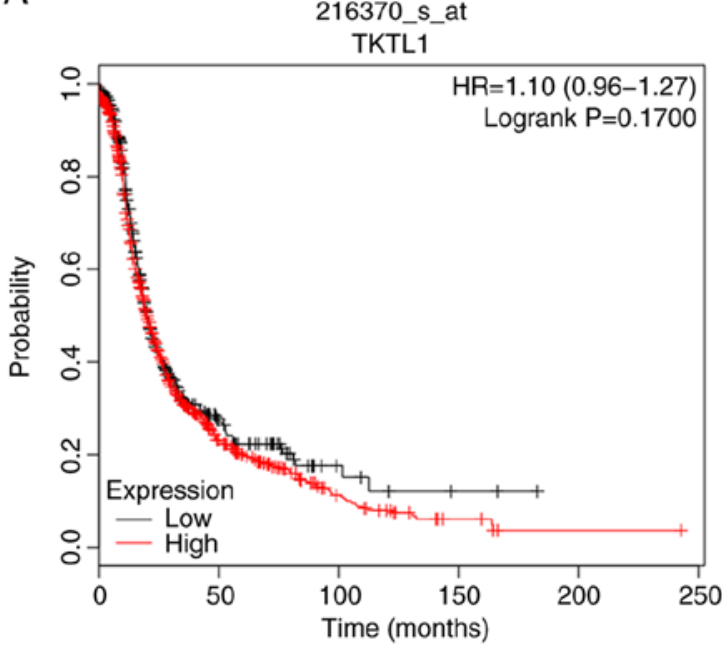

Number at risk

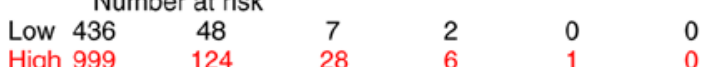

B

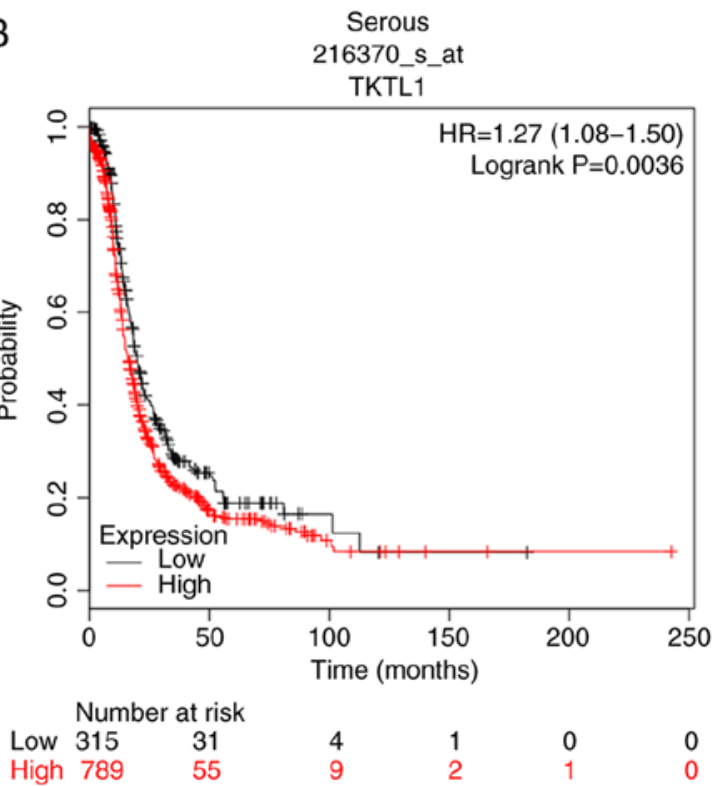

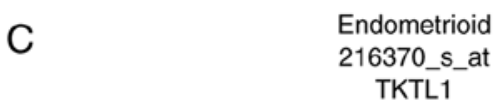

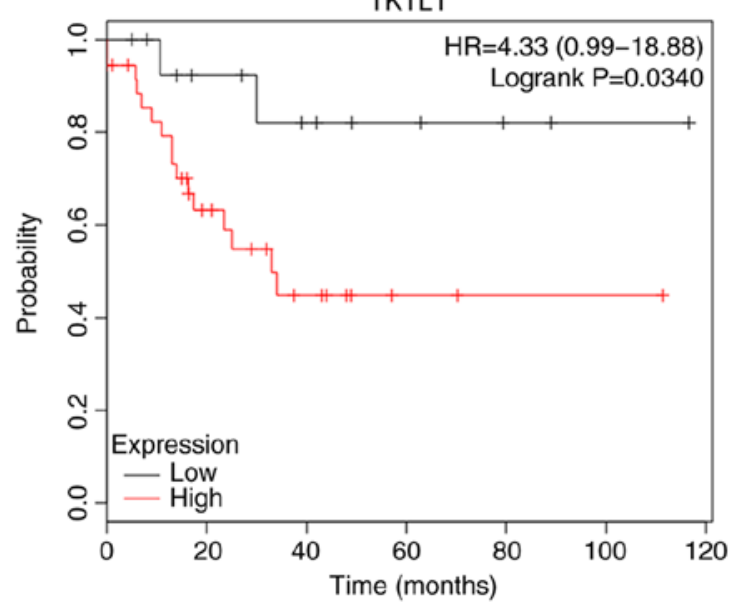

\begin{tabular}{ccccccc}
\multicolumn{2}{c}{ Number at risk } & & & & \\
Low 15 & 10 & 6 & 4 & 2 & 1 & 0 \\
High 36 & 16 & 8 & 2 & 1 & 1 & 0
\end{tabular}

Figure 4. Prognostic value of TKTL1 expression for PFS of patients with ovarian cancer based on the Kaplan-Meier plotter. Affymetrix identifier, 216370_s_at TKTL1. (A-C) PFS curves for $(A)$ all patients $(\mathrm{N}=1,435)$, $(\mathrm{B})$ patients with serous cancer $(\mathrm{N}=1,104)$ and $(\mathrm{C})$ patients with endometrioid cancer $(\mathrm{N}=51)$. TKTL1, transketolase-like 1; PFS, progression-free survival.

study is available concerning the prognostic values of TKT in ovarian cancer, which reported that patients with high nuclear TKT expression in peritoneal metastases exhibited shorter OS time compared with that of patients with low TKT protein levels (22). However, no correlation was identified between PFS and TKT expression in either primary cancer or peritoneal metastases (22). Thus, whether or not TKT mRNA has a prognostic role in patients with ovarian cancer remains to be determined. In the present study, a significant upregulation of TKT expression was observed in ovarian cancer compared with that in normal ovarian tissue. In addition, high TKT mRNA expression was associated with poor PFS in all patients with ovarian cancer, as well as in patients with serous-type ovarian cancer. Although the OS between the high and low-TKT expression groups in all ovarian cancer and serous ovarian cancer samples was not significantly different, the P-value was $<0.1$.

The mechanism underlying the carcinogenic roles of TKT in tumors has been investigated in a number of studies. A recent study has reported that TKT interacts with SH2 domain-containing 5 and activates STAT3 phosphorylation, thus promoting the proliferation of liver cancer cells (34). This result suggested that STAT3 may be involved in the regulation of liver cancer cell proliferation by TKT. Lin et al (35) demonstrated that the knockdown of TKT prevents vascular endothelial growth factor secretion and inhibits cell proliferation in lung cancer. To the best of our knowledge, no conclusive results are available on the mechanism of TKT in ovarian cancer, which needs further investigation. 
A

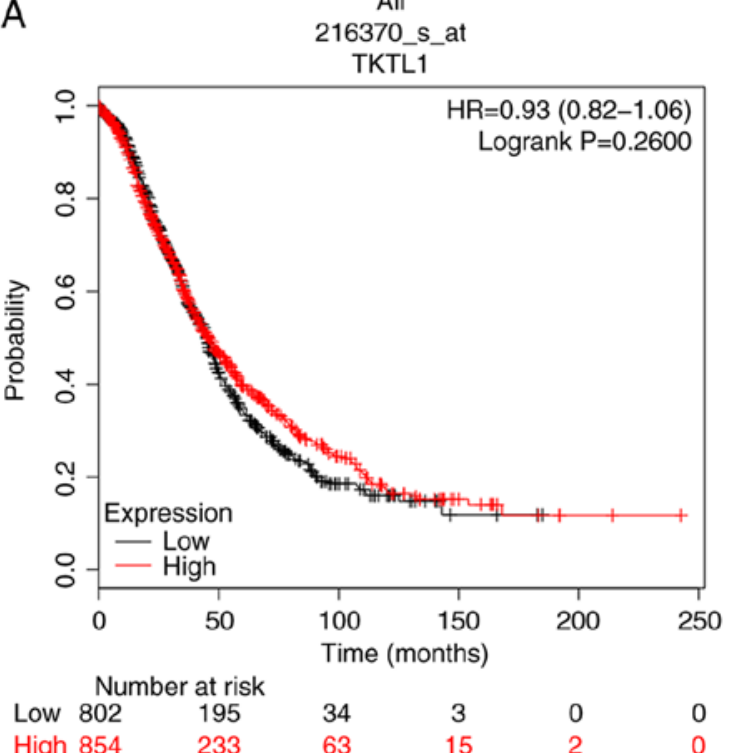

B

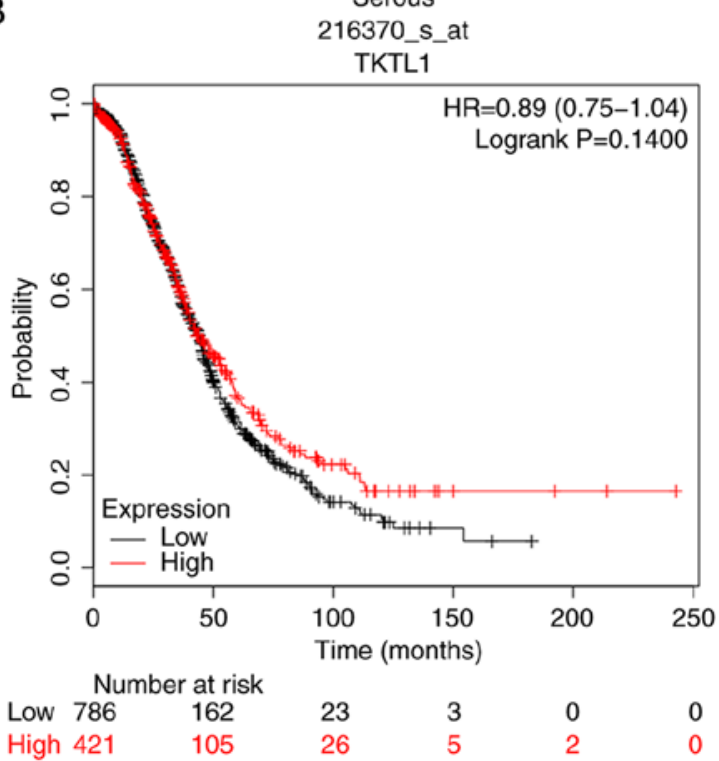

C 216370_s_at

TKTL1

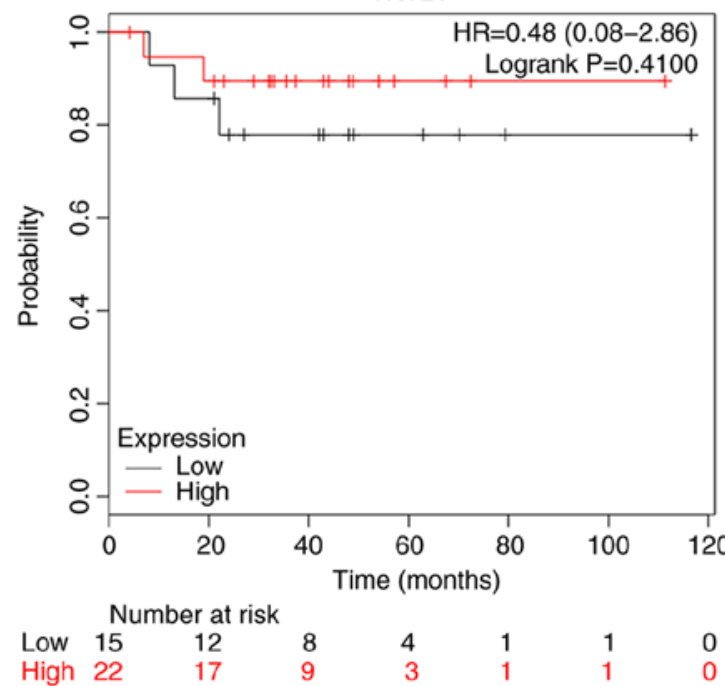

Figure 5. Prognostic value of TKTL1 expression for OS of patients with ovarian cancer based on the Kaplan-Meier plotter. Affymetrix identifier, 216370_s_at TKTL1. (A-C) OS curves for (A) all patients $(\mathrm{N}=1,656)$, (B) patients with serous cancer $(\mathrm{N}=1,207)$ and $(\mathrm{C})$ patients with endometrioid cancer $(\mathrm{N}=37)$. TKTL1, transketolase-like 1; OS, overall survival.

Previous studies have demonstrated that TKTL1 serves crucial roles in cancer prognosis. High expression levels of TKTL1 have been reported to be a significant indicator of poor outcome in colorectal (36) and gastric (18) cancer, esophageal squamous cell carcinoma (37), and locally advanced rectal (38) and non-small cell lung (17) cancer. In ovarian cancer, TKTL1 expression was observed in $81 \%$ of granulosa cell tumors of the ovary, but not in normal ovarian tissue (39). Krockenberger et al (40) also demonstrated that the expression of TKTL1 is significantly higher in tumor tissues of serous papillary ovarian adenocarcinomas compared with that in borderline tumors or normal tissues. In addition, increased expression of TKTL1 was associated with higher Federation International of Gynecology and Obstetrics stage, initial ascites and higher histopathological grade in serous papillary ovarian carcinoma (40). These results indicated that TKTL1 may be an oncogene in ovarian carcinogenesis and development.
However, to the best of our knowledge, no studies on the prognostic significance of TKTL1 in ovarian cancer are currently available. In the present study, upregulation of TKTL1 expression was demonstrated in all ovarian cancer cases, especially in serous ovarian cancer. In addition, high TKTL1 mRNA expression levels were significantly associated with poor PFS in patients with serous and endometrioid ovarian cancer. However, when the histological types of ovarian cancer were not distinguished, TKTL1 exhibited no effects on PFS in all patients with ovarian cancer due to the different cut-off values in different histological types. The cut-off values used for serous and endometrioid ovarian cancer in the present study were 10 and 24, respectively, which meant that patients with serous and endometrioid ovarian cancer were split differently to separate the high and low-expression groups. According to this divisional standard, patients with serous ovarian cancer with transcription level $>10$ comprised the high expression 


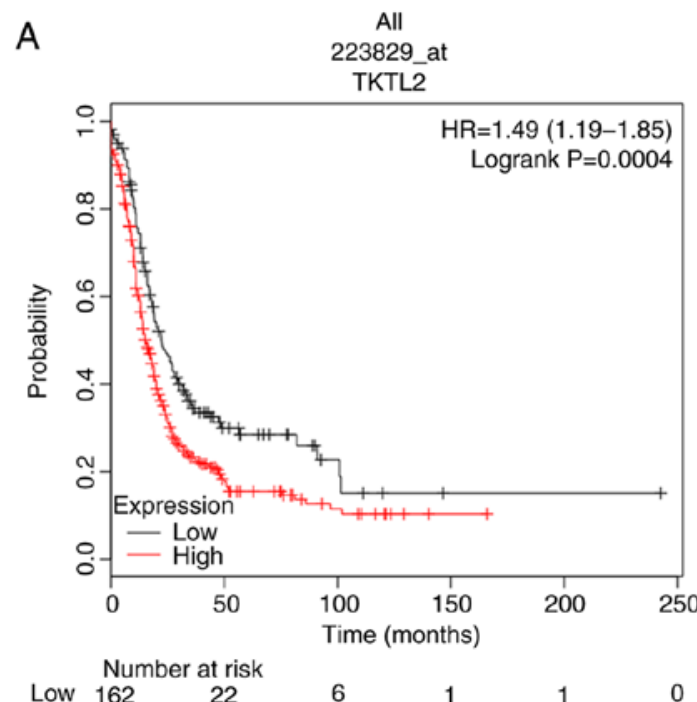

B Serous

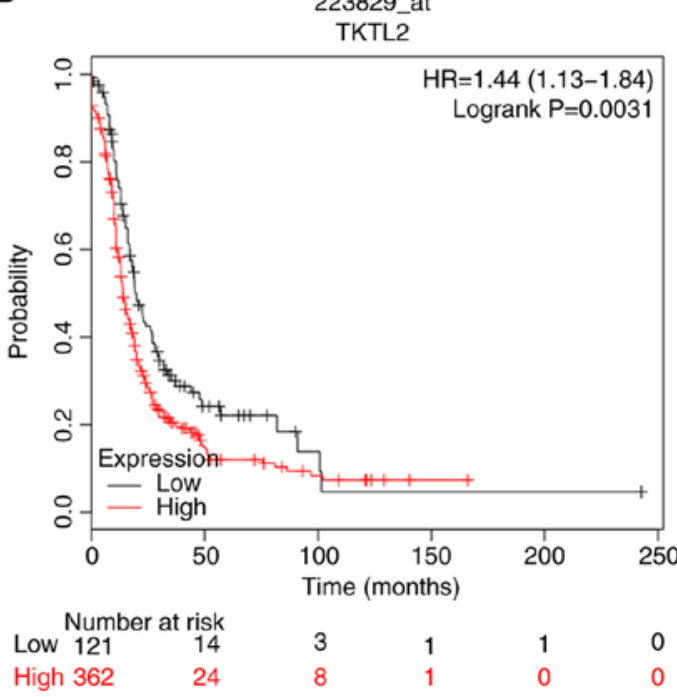

High 452 - 32

$10 \quad 1 \quad 000$

Endometrioid

223829_at
TKTL2

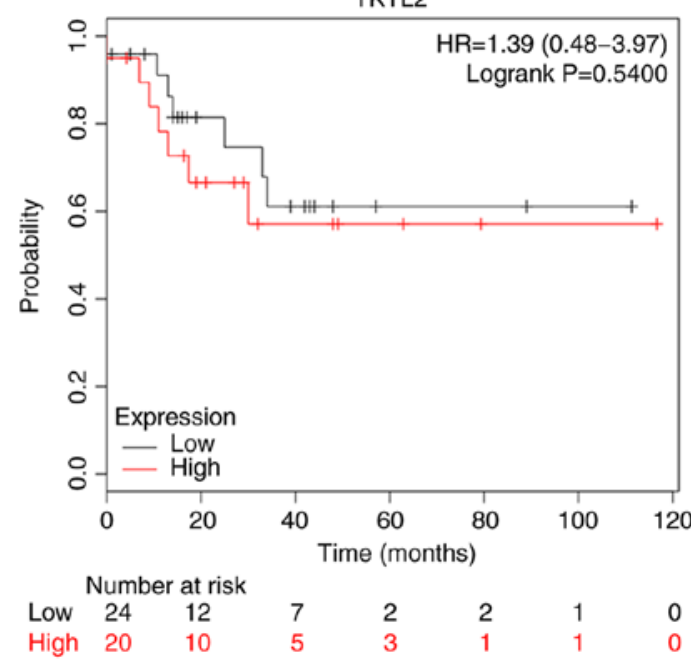

Figure 6. Prognostic value of TKTL2 expression for PFS of patients with ovarian cancer based on the Kaplan-Meier plotter. Affymetrix identifier, 223829 at TKTL2. (A-C) PFS curves for (A) all patients $(\mathrm{N}=614)$, (B) patients with serous cancer $(\mathrm{N}=483)$ and $(\mathrm{C})$ patients with endometrioid cancer $(\mathrm{N}=44)$. TKTL2, transketolase-like 2; PFS, progression-free survival.

group, whereas patients with serous ovarian cancer with transcription level $<10$ comprised the low expression group. In endometrioid ovarian cancer, patients whose expression value is $>24$ should be classified in the high expression group, whereas patients whose expression value is $<24$ should be classified in the low expression group. However, if this study used the same cut-off, such as 15 , to separate the high and low groups, ignoring the histological types of ovarian cancer, serous ovarian cancer patients with actually high expression would be mistakenly defined as low group, and endometrioid ovarian cancer patients with actually low expression would be mistakenly classified as high expression, thereby affecting the results of analysis. Therefore, the histology of ovarian cancer should be considered when assessing the prognostic roles of TKTL1 in ovarian cancer.

Studies on the function of TKTL2 in malignancies are limited. Chen et al (41) compared TKTL2 expression between uterine cervix cancer cells and normal human endocervical epithelial cells, and no significant differences between the two types of cell lines were observed. A similar result was observed in nasopharyngeal carcinoma (42). To the best of our knowledge, no studies on TKTL2 in ovarian cancer are currently available. In the present study, TKTL2 expression levels were compared between serous, endometrioid, clear cell or mucinous ovarian cancer tissues and normal ovarian tissues; however, no differences were observed. By contrast, high TKTL2 mRNA expression was significantly associated with poor PFS and OS in all patients with ovarian cancer. The negative result of the relative expression of TKTL2 in ovarian cancer may be due to the limited sample size, as the Lu dataset, which was used to compare the differential expression of TKTL2 between tumor and normal tissue, only included 5 normal ovarian tissue samples. Differential expression of TKTL2 between ovarian cancer and normal ovarian tissue requires further study.

The prognostic value of the transketolase family genes in ovarian cancer with different clinical stages was further 
A

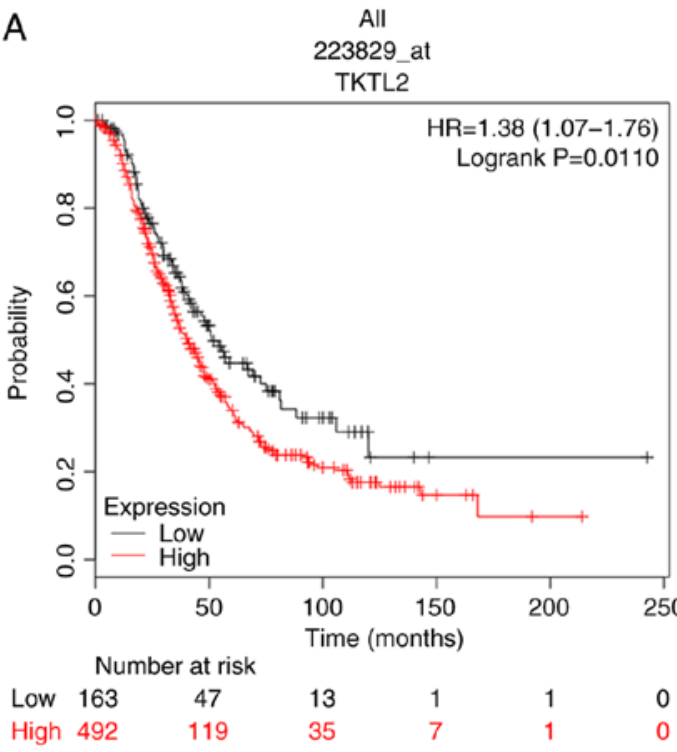

C
Serous
223829_at
TKTL2

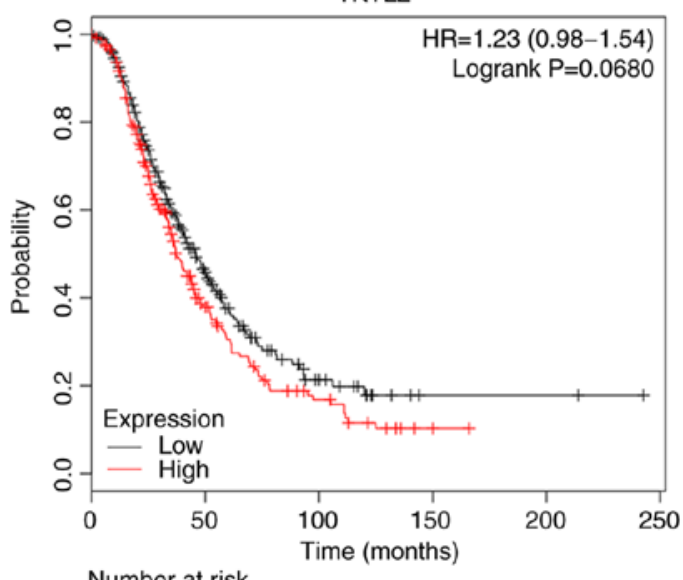

Number at risk

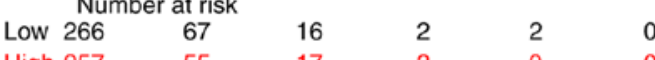

High 257

Endometrioid

223829_at

TKTL2

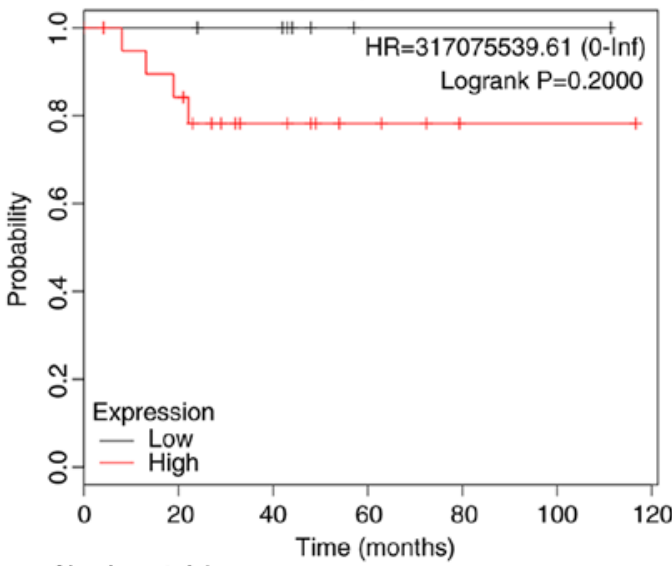

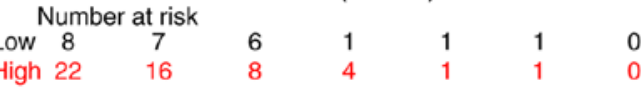

Figure 7. Prognostic value of TKTL2 expression for OS of patients with ovarian cancer based on the Kaplan-Meier plotter. Affymetrix identifier, 223829_at TKTL2. (A-C) OS curves for (A) all patients $(\mathrm{N}=655)$, (B) patients with serous cancer $(\mathrm{N}=523)$ and $(\mathrm{C})$ patients with endometrioid cancer (N=30), TKTL2, transketolase-like 2; OS, overall survival.
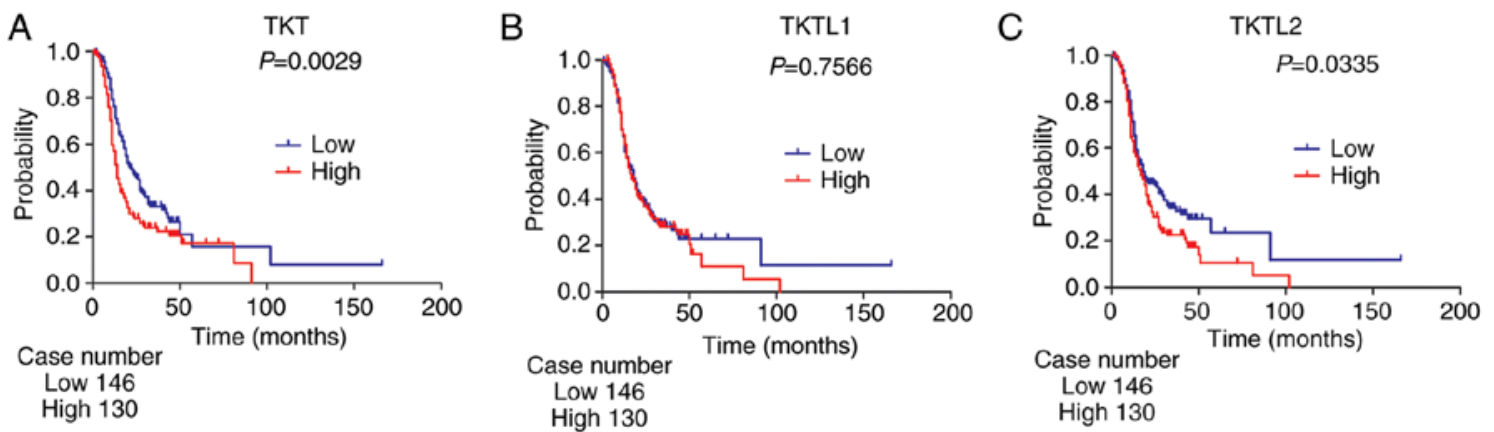

Figure 8. Prognostic value of TKT, TKTL1 and TKTL2 expression in all patients with ovarian cancer from the GSE9891 dataset. (A-C) Progression-free survival curves for (A) TKT, (B) TKTL1 and (C) TKTL2. TKT, transketolase; TKTL1, TKT-like 1; TKTL2, TKT-like 2.

investigated. The three transketolase genes were associated with poor PFS in patients with stage III/IV ovarian cancer, but exhibited no effects on PFS among patients with stage I/II ovarian cancer. These results suggested that the transketolase family genes may be a negative prognostic indicator for late-stage ovarian cancer. 
Table II. Association of transketolase family genes with progression-free survival in patients with different clinical stages of ovarian cancer.

\begin{tabular}{lcccc}
\hline Transketolase family gene & Clinical stage & Patients, $\mathrm{n}$ & HR (95\% CI) & P-value \\
\hline TKT & I+II & 163 & $0.75(0.42-1.34)$ & 0.3300 \\
& III+IV & 1,081 & $1.24(1.08-1.43)$ & $0.0025^{\mathrm{a}}$ \\
TKTL1 & I+II & 163 & $1.40(0.76-2.59)$ & 0.2800 \\
& III+IV & 1,081 & $1.32(1.12-1.56)$ & $0.0008^{\mathrm{a}}$ \\
TKTL2 & I+II & 115 & $2.06(0.84-5.08)$ & 0.1100 \\
& III+IV & 494 & $1.36(1.08-1.70)$ & $0.0079^{\mathrm{a}}$
\end{tabular}

${ }^{\mathrm{a}} \mathrm{P}<0.05$. TKT, transketolase; TKTL1, TKT-like 1; TKTL2, TKT-like 2; HR, hazard ratio; CI, confidence interval.

Table III. Association of transketolase family genes with progression-free survival in patients with different pathological grades of ovarian cancer.

\begin{tabular}{lcccc}
\hline Transketolase family gene & Pathological grade & Patients, $\mathrm{n}$ & HR (95\% CI) & P-value \\
\hline TKT & I+II & 293 & $1.46(1.09-1.96)$ & $0.0120^{\mathrm{a}}$ \\
& III & 837 & $1.21(1.03-1.43)$ & $0.0220^{\mathrm{a}}$ \\
TKTL1 & I+II & 293 & $1.41(1.04-1.91)$ & $0.0250^{\mathrm{a}}$ \\
& III & 837 & $1.37(1.12-1.67)$ & $0.0020^{\mathrm{a}}$ \\
TKTL2 & I+II & 189 & $1.53(1.03-2.28)$ & $0.0350^{\mathrm{a}}$ \\
& III & 315 & $1.51(1.18-1.95)$ & $0.0011^{\mathrm{a}}$ \\
\hline
\end{tabular}

${ }^{\mathrm{a}}<<0.05$. TKT, transketolase; TKTL1, TKT-like 1; TKTL2, TKT-like 2; HR, hazard ratio; CI, confidence interval.

Table IV. Association of transketolase family genes with progression-free survival in patients with ovarian cancer who received chemotherapy.

\begin{tabular}{llccr}
\hline Transketolase family gene & Chemotherapy & Patients, $\mathrm{n}$ & HR $(95 \% \mathrm{CI})$ & P-value \\
\hline TKT & Platinum & 1,259 & $1.19(1.05-1.36)$ & $0.0078^{\mathrm{a}}$ \\
& Taxol & 715 & $1.16(0.95-1.40)$ & 0.1400 \\
& Taxol + platinum & 698 & $1.14(0.94-1.39)$ & 0.1900 \\
TKTL1 & Platinum & 1,259 & $0.91(0.80-1.04)$ & 0.1500 \\
& Taxol & 715 & $1.23(1.03-1.48)$ & $0.0250^{\mathrm{a}}$ \\
& Taxol + platinum & 698 & $1.24(1.03-1.50)$ & $0.0240^{\mathrm{a}}$ \\
TKTL2 & Platinum & 502 & $1.31(1.08-1.59)$ & $0.0063^{\mathrm{a}}$ \\
& Taxol & 381 & $1.44(1.13-1.84)$ & $0.0031^{\mathrm{a}}$ \\
& Taxol + platinum & 380 & $1.44(1.13-1.83)$ & $0.0034^{\mathrm{a}}$ \\
\hline
\end{tabular}

aP $<0.05$. TKT, transketolase; TKTL1, TKT-like 1; TKTL2, TKT-like 2; HR, hazard ratio; CI, confidence interval.

Platinum and taxol are the two most frequently used chemotherapeutic drugs for treating ovarian cancer (43). However, a number of patients ultimately progress to recurrent, chemotherapy-resistant tumors, and the majority of these patients succumb to the disease within one year (44). There is a great need to identify prognostic markers to predict the outcome of patients with ovarian cancer treated with platinum and paclitaxel. The results of the present study demonstrated that the transketolase family genes were associated with chemotherapy sensitivity in ovarian cancer. Transketolase family genes served different roles in chemotherapy sensitivity. The results revealed that enhanced TKT expression was associated with unfavorable PFS in patients with ovarian cancer treated with platinum, whereas high TKTL1 expression was associated with poor PFS in patients treated with taxol. TKTL2 was associated with poor outcomes in patients treated with platinum plus taxol. These results were consistent with those from previous studies. Taoka et al (45) 
Table V. Association of transketolase family genes with progression-free survival in patients with ovarian cancer with different TP53 mutation status.

\begin{tabular}{lcrcl}
\hline $\begin{array}{l}\text { Transketolase } \\
\text { family gene }\end{array}$ & $\begin{array}{c}\text { TP53 } \\
\text { mutation }\end{array}$ & Patients, & HR $(95 \% \mathrm{CI})$ & P-value \\
\hline TKT & No & 84 & $0.59(0.32-1.07)$ & 0.0780 \\
& Yes & 483 & $1.53(1.22-1.92)$ & $0.0002^{\mathrm{a}}$ \\
TKTL1 & No & 84 & $1.59(0.91-2.79)$ & 0.1000 \\
& Yes & 483 & $1.37(1.07-1.75)$ & $0.0130^{\mathrm{a}}$ \\
TKTL2 & No & 19 & $2.03(0.64-6.38)$ & 0.2200 \\
& Yes & 124 & $1.88(1.24-2.86)$ & $0.0027^{\mathrm{a}}$ \\
\hline
\end{tabular}

${ }^{\text {aP }}<0.05$. TP53, tumor protein $\mathrm{p} 53$; HR, hazard ratio; CI, confidence interval; TKT, transketolase; TKTL1, TKT-like 1; TKTL2, TKT-like 2.

compared differential protein expression profiles between platinum-naïve and platinum-resistant bladder cancer cell lines to identify the candidate molecules associated with platinum resistance, and revealed that the expression of TKT was increased by 1.5-fold in platinum-resistant bladder cancer compared with that in platinum-naïve cells. Yang et al (31) also demonstrated that silencing of TKT significantly reduced cell viability and increased apoptosis in the presence of platinum via glutathione depletion and reactive oxygen species generation in cervical cancer. In addition, overexpression of TKT increased resistance to platinum chemotherapy by increasing cell viability and decreasing apoptosis in cervical cancer in vitro (31). A study on the role of TKTL1 in taxol sensitivity revealed that the inhibition of TKTL1 significantly decreased the proliferation rate and the $\mathrm{IC}_{50}$ of taxol in ovarian cancer (46). Mechanistically, following TKTL1 knockdown, $\mathrm{NADPH}$ levels were reduced and $\mathrm{NADP}^{+}$levels were increased compared with the levels in the control group, which led to taxol sensitivity in ovarian cancer (46).

The TP53 gene, located on chromosome 17p13, encodes the tumor-suppressor protein p53 (47). TP53 serves an important role in the regulation of the cell cycle, DNA repair and cell death through apoptosis (48). Mutation or loss of function of the TP53 gene is a frequent and important genetic alteration in the development of ovarian cancer (49). Harami-Papp et al (50) compared gene expression between TP53-mutated and wild-type tissue samples from patients with breast cancer and revealed that transketolase was upregulated in TP53-mutated breast cancer compared with its expression in TP53 wild-type breast cancer. In the present study, the prognostic roles of individual transketolase genes in patients with ovarian cancer with different TP53 mutation status were examined; the three transketolase family genes were associated with poor PFS in patients with TP53-mutated, but not with TP53 wild-type, ovarian cancer. Therefore, the transketolase family may have an effect on the prognosis of TP53-mutated ovarian cancer.

A lack of experimental data was the main limitation of the present study. To strengthen the conclusions, cell and animal models with transketolase gene knockdown and overexpression may be constructed, which would verify the tumorigenic functions of transketolase genes in ovarian cancer. Future studies may also collect clinical information and perform immunohistochemistry on clinical cervical cancer samples to subsequently validate the prognostic roles of transketolase genes.

In summary, the present study identified elevated TKT and TKTL1 expression in ovarian cancer compared with that in normal ovarian tissue. High mRNA expression of the above three transketolase genes was demonstrated to be associated with poor PFS in patients with serous ovarian cancer, especially those at an advanced clinical stage. TKTL2 was also significantly associated with poor OS in all patients with ovarian cancer. In addition, transketolase family genes served a role in predicting PFS in patients with ovarian cancer treated with platinum and/or taxol, as well as in patients with TP53-mutated ovarian cancer. These results suggest that transketolase genes have a prognostic value in ovarian cancer. This may facilitate better prognostic stratification and may be useful for the detailed understanding of the complexity and heterogeneity implicated in the molecular biology of ovarian cancer.

\section{Acknowledgements}

Not applicable.

\section{Funding}

The present study was supported by the Key Laboratory of Wenzhou City-Gynecological Oncology (grant no.ZD201603). The sponsor had no involvement in the collection, analysis and interpretation of data or in the writing of the manuscript.

\section{Availability of data and materials}

The datasets generated and/or analyzed during the present study are available in Oncomine (https://www. oncomine.org/resource/login.html) and the Kaplan-Meier plotter database (http://www.kmplot.com/analysis/index. $\mathrm{php}$ ? $=$ service $\&$ cancer $=$ ovar $)$.

\section{Authors' contributions}

MZ and XZ conceived and designed the study. MY and JZ collected the data. MZ and MY analyzed the data. All authors participated in manuscript writing. All authors contributed to data analysis, drafting and revising the manuscript, gave final approval of the version to be published and agreed to be accountable for all aspects of the work.

\section{Ethics approval and consent to participate}

All data in the present study were obtained from publicly available datasets. No additional patients were enrolled in the study.

\section{Patient consent for publication}

Not applicable.

\section{Competing interests}

The authors declare that they have no competing interests. 


\section{References}

1. Siegel RL, Miller KD and Jemal A: Cancer statistics, 2019. CA Cancer J Clin 69: 7-34, 2019.

2. Coleman MP, Forman D, Bryant H, Butler J, Rachet B, Maringe C, Nur U, Tracey E, Coory M, Hatcher J, et al: Cancer survival in Australia, Canada, Denmark, Norway, Sweden and the UK, 1995-2007 (the International Cancer Benchmarking Partnership): An analysis of population-based cancer registry data. Lancet 377: 127-138, 2011.

3. Warburg O: On the origin of cancer cells. Science 123: 309-314, 1956.

4. Ward PS and Thompson CB: Metabolic reprogramming: A cancer hallmark even warburg did not anticipate. Cancer Cell 21: 297-308, 2012.

5. Zheng Y, Huang Q, Ding Z, Liu T, Xue C, Sang X and Gu J: Genome-wide DNA methylation analysis identifies candidate epigenetic markers and drivers of hepatocellular carcinoma. Brief Bioinform 19: 101-108, 2018.

6. Diaz-Moralli S, Tarrado-Castellarnau M, Alenda C, Castells A and Cascante M: Transketolase-like 1 expression is modulated during colorectal cancer progression and metastasis formation. PLoS One 6: e25323, 2011.

7. Kowalik MA, Columbano A and Perra A: Emerging role of the pentose phosphate pathway in hepatocellular carcinoma. Front Oncol 7: 87, 2017.

8. Jiang P, Du W and Wu M: Regulation of the pentose phosphate pathway in cancer. Protein Cell 5: 592-602, 2014.

9. Boros LG, Puigjaner J, Cascante M, Lee WN, Brandes JL, Bassilian S, Yusuf FI, Williams RD, Muscarella P, Melvin WS and Schirmer WJ: Oxythiamine and dehydroepiandrosterone inhibit the nonoxidative synthesis of ribose and tumor cell proliferation. Cancer Res 57: 4242-4248, 1997.

10. Coy JF, Dressler D, Wilde J and Schubert P: Mutations in the transketolase-like gene TKTL1: Clinical implications for neurodegenerative diseases, diabetes and cancer. Clin Lab 51: 257-273, 2005.

11. Zhao J and Zhong CJ: A review on research progress of transketolase. Neurosci Bull 25: 94-99, 2009.

12. Li J, Zhu SC, Li SG, Zhao Y, Xu JR and Song CY: TKTL1 promotes cell proliferation and metastasis in esophageal squamous cell carcinoma. Biomed Pharmacother 74: 71-76, 2015.

13. Fritz P, Coy JF, Murdter TE, Ott G, Alscher MD and Friedel G: TKTL-1 expression in lung cancer. Pathol Res Pract 208: 203-209, 2012

14. Sun W, Liu Y, Glazer CA, Shao C, Bhan S, Demokan S, Zhao M, Rudek MA, Ha PK and Califano JA: TKTL1 is activated by promoter hypomethylation and contributes to head and neck squamous cell carcinoma carcinogenesis through increased aerobic glycolysis and HIFlalpha stabilization. Clin Cancer Res 16: 857-866, 2010.

15. Krockenberger M, Engel JB, Schmidt M, Kohrenhagen N, Hausler SF, Dombrowski Y, Kapp M, Dietl J and Honig A: Expression of transketolase-like 1 protein (TKTL1) in human endometrial cancer. Anticancer Res 30: 1653-1659, 2010.

16. Chao YK, Peng TL, Chuang WY, Yeh CJ, Li YL, Lu YC and Cheng AJ: Transketolase serves a poor prognosticator in esophageal cancer by promoting cell Invasion via epithelial-mesenchymal transition. J Cancer 7: 1804-1811, 2016.

17. Kayser G, Sienel W, Kubitz B, Mattern D, Stickeler E, Passlick B, Werner $\mathrm{M}$ and Zur Hausen A: Poor outcome in primary non-small cell lung cancers is predicted by transketolase TKTL1 expression. Pathology 43: 719-724, 2011.

18. Song Y, Liu D and He G: TKTL1 and p63 are biomarkers for the poor prognosis of gastric cancer patients. Cancer Biomark 15: 591-597, 2015

19. Benito A, Polat IH, Noe V, Ciudad CJ, Marin S and Cascante M Glucose-6-phosphate dehydrogenase and transketolase modulate breast cancer cell metabolic reprogramming and correlate with poor patient outcome. Oncotarget 8: 106693-106706, 2017.

20. Langbein S, Zerilli M, Zur Hausen A, Staiger W, Rensch-Boschert K, Lukan N, Popa J, Ternullo MP, Steidler A, Weiss C, et al: Expression of transketolase TKTL1 predicts colon and urothelial cancer patient survival: Warburg effect reinterpreted. Br J Cancer 94: 578-585, 2006.

21. Bery A, Leung F, Smith CR, Diamandis EP and Kulasingam V: Deciphering the ovarian cancer ascites fluid peptidome. Clin Proteomics 11: 13, 2014.
22. Ricciardelli C, Lokman NA, Cheruvu S, Tan IA, Ween MP, Pyragius CE, Ruszkiewicz A, Hoffmann P and Oehler MK: Transketolase is upregulated in metastatic peritoneal implants and promotes ovarian cancer cell proliferation. Clin Exp Metastasis 32: 441-455, 2015.

23. Rhodes DR, Kalyana-Sundaram S, Mahavisno V, Varambally R, Yu J, Briggs BB, Barrette TR, Anstet MJ, Kincead-Beal C, Kulkarni P, et al: Oncomine 3.0: Genes, pathways, and networks in a collection of 18,000 cancer gene expression profiles. Neoplasia 9: 166-180, 2007

24. Gyorffy B, Lanczky A, Eklund AC, Denkert C, Budczies J, Li Q and Szallasi Z: An online survival analysis tool to rapidly assess the effect of 22,277 genes on breast cancer prognosis using microarray data of 1,809 patients. Breast Cancer Res Treat 123: 725-731, 2010.

25. Gyorffy B, Surowiak P, Budczies J and Lanczky A: Online survival analysis software to assess the prognostic value of biomarkers using transcriptomic data in non-small-cell lung cancer. PLoS One 8: e82241, 2013.

26. Gyorffy B, Lanczky A and Szallasi Z: Implementing an online tool for genome-wide validation of survival-associated biomarkers in ovarian-cancer using microarray data from 1287 patients. Endocr Relat Cancer 19: 197-208, 2012.

27. Zhao M, Li S, Zhou L, Shen Q, Zhu H and Zhu X: Prognostic values of excision repair cross-complementing genes mRNA expression in ovarian cancer patients. Life Sci 194: 34-39, 2018.

28. Tian X, Han Y, Yu L, Luo B, Hu Z, Li X, Yang Z, Wang X, Huang W, Wang H, et al: Decreased expression of ALDH5A1 predicts prognosis in patients with ovarian cancer. Cancer Biol Ther 18: 245-251, 2017.

29. Tothill RW, Tinker AV, George J, Brown R, Fox SB, Lade S, Johnson DS, Trivett MK, Etemadmoghadam D, Locandro B, et al: Novel molecular subtypes of serous and endometrioid ovarian cancer linked to clinical outcome. Clin Cancer Res 14: 5198-5208, 2008.

30. Tseng CW, Kuo WH, Chan SH, Chan HL, Chang KJ and Wang LH: Transketolase regulates the metabolic switch to control breast cancer cell metastasis via the $\alpha$-Ketoglutarate signaling pathway. Cancer Res 78: 2799-2812, 2018.

31. Yang $\mathrm{H}$, Wu XL, Wu KH, Zhang R, Ju LL, Ji Y, Zhang YW, Xue SL, Zhang YX, Yang YF and Yu MM: MicroRNA-497 regulates cisplatin chemosensitivity of cervical cancer by targeting transketolase. Am J Cancer Res 6: 2690-2699, 2016

32. Chen Y, Xiong X, Wang Y, Zhao J, Shi H, Zhang H, Wang Y, Wei Y, Xue W and Zhang J: Proteomic screening for serum biomarkers for cervical cancer and their clinical significance. Med Sci Monit 25: 288-297, 2019.

33. Yi H, Zheng X, Song J, Shen R, Su Y and Lin D: Exosomes mediated pentose phosphate pathway in ovarian cancer metastasis: A proteomics analysis. Int J Clin Exp Pathol 8: 15719-15728, 2015.

34. Zheng Y, Ming P, Zhu C, Si Y, Xu S, Chen A, Wang J and Zhang B: Hepatitis B virus $\mathrm{X}$ protein-induced $\mathrm{SH} 2$ domain-containing 5 (SH2D5) expression promotes hepatoma cell growth via an SH2D5-transketolase interaction. J Biol Chem 294: 4815-4827, 2019.

35. Lin CC, Chen LC, Tseng VS, Yan JJ, Lai WW, Su WP, Lin CH, Huang CY and Su WC: Malignant pleural effusion cells show aberrant glucose metabolism gene expression. Eur Respir J 37: 1453-1465, 2011.

36. Ahopelto K, Bockelman C, Hagstrom J, Koskensalo S and Haglund C: Transketolase-like protein 1 expression predicts poor prognosis in colorectal cancer. Cancer Biol Ther 17: 163-168, 2016.

37. Shi Z, Tang Y, Li K and Fan Q: TKTL1 expression and its downregulation is implicated in cell proliferation inhibition and cell cycle arrest in esophageal squamous cell carcinoma. Tumour Biol 36: 8519-8529, 2015.

38. Schwaab J, Horisberger K, Ströbel P, Bohn B, Gencer D, Kähler G, Kienle P, Post S, Wenz F, Hofmann WK, et al: Expression of Transketolase like gene 1 (TKTL1) predicts disease-free survival in patients with locally advanced rectal cancer receiving neoadjuvant chemoradiotherapy. BMC Cancer 11: 363, 2011.

39. Schmidt M, Kammerer U, Segerer S, Cramer A, Kohrenhagen N, Dietl J and Voelker HU: Glucose metabolism and angiogenesis in granulosa cell tumors of the ovary: Activation of Akt, expression of M2PK, TKTL1 and VEGF. Eur J Obstet Gynecol Reprod Biol 139: 72-78, 2008.

40. Krockenberger M, Honig A, Rieger L, Coy JF, Sutterlin M, Kapp M, Horn E, Dietl J and Kammerer U: Transketolase-like 1 expression correlates with subtypes of ovarian cancer and the presence of distant metastases. Int J Gynecol Cancer 17: 101-106, 2007. 
41. Chen H, Yue JX, Yang SH, Ding H, Zhao RW and Zhang S: Overexpression of transketolase-like gene 1 is associated with cell proliferation in uterine cervix cancer. J Exp Clin Cancer Res 28: 43, 2009.

42. Zhang S, Yang JH and Cai PC: Effects of transketolase-like gene TKTL1 on occurrence and metastasis of human nasopharyngeal carcinoma. Zhonghua Yi Xue Za Zhi 88: 3131-3134, 2008 (In Chinese)

43. Ge L, Li N, Yuan GW, Sun YC and Wu LY: Nedaplatin and paclitaxel compared with carboplatin and paclitaxel for patients with platinum-sensitive recurrent ovarian cancer. Am J Cancer Res 8: 1074-1082, 2018.

44. Griffiths RW, Zee YK, Evans S, Mitchell CL, Kumaran GC, Welch RS, Jayson GC, Clamp AR and Hasan J: Outcomes after multiple lines of chemotherapy for platinum-resistant epithelial cancers of the ovary, peritoneum, and fallopian tube. Int J Gynecol Cancer 21: 58-65, 2011.

45. Taoka Y, Matsumoto K, Ohashi K, Minamida S, Hagiwara M, Nagi S, Saito T, Kodera Y and Iwamura M: Protein expression profile related to cisplatin resistance in bladder cancer cell lines detected by two-dimensional gel electrophoresis. Biomed Res 36: 253-261, 2015.
46. Zheng $\mathrm{X}$ and Li H: TKTL1 modulates the response of paclitaxel-resistant human ovarian cancer cells to paclitaxel. Biochem Biophys Res Commun 503: 572-579, 2018.

47. Na K, Sung JY and Kim HS: TP53 Mutation status of tubo-ovarian and peritoneal high-grade serous carcinoma with a wild-type p53 immunostaining pattern. Anticancer Res 37: 6697-6703, 2017.

48. Kalamanathan S, Bates V, Lord R and Green JA: The mutational profile of sporadic epithelial ovarian carcinoma. Anticancer Res 31: 2661-2668, 2011.

49. Munksgaard PS and Blaakaer J: The association between endometriosis and ovarian cancer: A review of histological, genetic and molecular alterations. Gynecol Oncol 124: 164-169, 2012

50. Harami-Papp H, Pongor LS, Munkacsy G, Horvath G, Nagy AM, Ambrus A, Hauser P, Szabo A, Tretter L and Gyorffy B: TP53 mutation hits energy metabolism and increases glycolysis in breast cancer. Oncotarget 7: 67183-67195, 2016.

(i) (9) This work is licensed under a Creative Commons Attribution-NonCommercial-NoDerivatives 4.0 International (CC BY-NC-ND 4.0) License. 